Article

\title{
The Transcription Factor Nfix Requires RhoA-ROCK1 Dependent Phagocytosis to Mediate Macrophage Skewing during Skeletal Muscle Regeneration
}

\author{
Marielle Saclier ${ }^{\circledR}$, Michela Lapi, Chiara Bonfanti, Giuliana Rossi ${ }^{+} \mathbb{D}^{\circ}$, Stefania Antonini \\ and Graziella Messina *(D) \\ Department of Biosciences, University of Milan, via Celoria 26, 20133 Milan, Italy; \\ marielle.saclier@unimi.it (M.S.); michela.lapi@unimi.it (M.L.); chiara.bonfanti@unimi.it (C.B.); \\ giuliana.rossi@epfl.ch (G.R.); stefania.antonini@unimi.it (S.A.) \\ * Correspondence: graziella.messina@unimi.it; Tel.: +3902 50314800 \\ + Current affiliation: Laboratory of Stem Cell Bioengineering, Institute of Bioengineering, School of Life Sciences \\ and School of Engineering, École Polytechnique Fédérale de Lausanne (EPFL), \\ 1015 Lausanne, Vaud, Switzerland.
}

Received: 19 February 2020; Accepted: 12 March 2020; Published: 13 March 2020

\begin{abstract}
Macrophages (MPs) are immune cells which are crucial for tissue repair. In skeletal muscle regeneration, pro-inflammatory cells first infiltrate to promote myogenic cell proliferation, then they switch into an anti-inflammatory phenotype to sustain myogenic cells differentiation and myofiber formation. This phenotypical switch is induced by dead cell phagocytosis. We previously demonstrated that the transcription factor Nfix, a member of the nuclear factor I (Nfi) family, plays a pivotal role during muscle development, regeneration and in the progression of muscular dystrophies. Here, we show that Nfix is mainly expressed by anti-inflammatory macrophages. Upon acute injury, mice deleted for Nfix in myeloid line displayed a significant defect in the process of muscle regeneration. Indeed, Nfix is involved in the macrophage phenotypical switch and macrophages lacking Nfix failed to adopt an anti-inflammatory phenotype and interact with myogenic cells. Moreover, we demonstrated that phagocytosis induced by the inhibition of the RhoA-ROCK1 pathway leads to Nfix expression and, consequently, to acquisition of the anti-inflammatory phenotype. Our study identified Nfix as a link between RhoA-ROCK1-dependent phagocytosis and the MP phenotypical switch, thus establishing a new role for Nfix in macrophage biology for the resolution of inflammation and tissue repair.
\end{abstract}

Keywords: macrophages; Nfix; skeletal muscle; phagocytosis; RhoA-ROCK1

\section{Introduction}

During their lifetime, tissues encounter physiological and non-physiological damages and an effective regeneration is necessary to make these tissues able to continuously sustain their biological functions. Macrophage-mediated inflammation is a fundamental step for tissue recovery. Macrophages (MPs) are immune cells required for tissue regeneration, as their depletion prevents regeneration of different tissues/organs, such as liver [1], spinal cord [2] and skeletal muscle [3]. In several regenerative processes, two populations of MPs have been described. The first population reaching the damaged tissue is the pro-inflammatory population, also called M1 MPs. Pro-inflammatory MPs secrete pro-inflammatory molecules, being the main actors of dead cell clearance. The second population is composed of the anti-inflammatory MPs, named M2 MPs, that come from pro-inflammatory MPs and are involved in the resolution of the inflammation, wound healing and tissue regeneration or repair [4-7]. Several studies have shown that an impaired or a precocious phenotypical switch 
from M1 to M2 MPs results in defective tissue regeneration [1,8,9]. Interestingly, it has been observed that phagocytosis is at the basis of the pro to anti-inflammatory phenotypical switch in MPs [3,8,10-13]. Although the process of the induction of phagocytosis is well-known ("find-me", "eat-me" and "don't eat-me" signals), the molecular and transcriptional pathways between phagocytosis and the phenotypical switch are still unexplored [10,11,14,15].

Interestingly, the interplay between MPs and tissue regeneration has been widely documented in skeletal muscle [7,16-20]. In vertebrates, muscle progenitors originate from pre-somitic and cranial mesoderm. In pre-natal period, two myogenic waves are necessary for muscle establishment: the first forms the basic muscle pattern and is called primary or "embryonic" myogenesis, while the second or "fetal" myogenesis is characterized by muscle maturation and growth [21]. Adult skeletal muscle is able to regenerate thanks to resident stem cells called satellite cells (SCs), located under the basal lamina of myofibers [22]. Upon injury, SCs exit from quiescence, proliferate, differentiate in myoblasts and fuse to reform myofibers [23]. Nuclear factor I X (Nfix) is a transcription factor belonging to the highly conserved DNA-binding nuclear factor one family (Nfi) together with Nfia, Nfib and Nfic [24]. Nfix has a key role in prenatal myogenesis by driving the transcriptional switch from embryonic to fetal myogenesis $[25,26]$. Nfix is also required for adult myogenesis upon injury, since its absence leads to defect of SC differentiation [27]. Finally, we recently demonstrated that the deletion of Nfix in two mouse models of muscular dystrophy induces a significant morphological and functional amelioration of the pathology by slowing-down muscle regeneration and promoting a switch towards a more oxidative musculature [28].

During muscle regeneration, myogenic cells and MPs closely interact [7]. Soon after injury, activated SCs attract blood monocytes that infiltrate damaged muscle and differentiate in pro-inflammatory MPs that stimulate the proliferation of myoblasts. Then, by removing dead cells, MPs switch to an anti-inflammatory phenotype that sustains myogenic differentiation [3,29]. While MPs are required for muscle regeneration, preventing MPs infiltration in dystrophic disease decreases muscle damage [30]. Thus, depending on a context of acute or chronic injury, MPs adopt a complete opposite function toward muscle cells and environment [31,32].

In this study, we address the role of Nfix in MPs during skeletal muscle regeneration, by using a mouse model in which Nfix is deleted specifically in MPs. We report that mice lacking Nfix in myeloid lineages exhibit a delay of muscle regeneration upon acute injury. We demonstrated that the RhoA-ROCK1-dependent phagocytosis induces Nfix, whose expression is necessary for the acquisition of anti-inflammatory phenotype and thus pro-regenerative properties through myogenic cells. Indeed, during the process of muscle regeneration, in the absence of Nfix, MPs are able to phagocyte, but failed to adopt an anti-inflammatory phenotype necessary for the resolution of inflammation and muscle regeneration.

\section{Materials and Methods}

\subsection{Animal Models and In Vivo Experimentations}

WT, Nfix ${ }^{\mathrm{fl} / \mathrm{fl}}$ and LysM ${ }^{\mathrm{CRE}}: \mathrm{Nfix}{ }^{\mathrm{fl} / \mathrm{fl}}$ mice were used in this study. $\mathrm{LysM}^{\mathrm{CRE}}: \mathrm{Nfix}{ }^{\mathrm{fl} / \mathrm{fl}}$ mice were generated, crossing Nfix ${ }^{\mathrm{fl} / \mathrm{fl}}$ mice obtained from Prof. Richard M. Gronostajski [33] and LysM $\mathrm{M}^{\mathrm{CRE}}$ mice obtained from Dr. Rémi Mounier [8]. All LysM ${ }^{\mathrm{CRE}}: \mathrm{Nfix}{ }^{\mathrm{fl} / \mathrm{fl}}$ mice analyzed were heterozygous for the LysM ${ }^{\mathrm{CRE}}$. Muscle regeneration was realized by the injection of $20 \mathrm{uL}$ of $100 \mathrm{uM}$ cardiotoxin (CTX, Latoxan, L8102) in the Tibialis anterior (TA) of 2-month-old mice. For the in vivo analysis of satellite cells and myoblasts proliferation, EdU (5-ethynyl-2'-deoxyuridine) was injected in Nfix ${ }^{\mathrm{fl} / \mathrm{fl}}$ and LysM ${ }^{C R E}: N f i x x^{\mathrm{fl} / \mathrm{fl}}$ mice in intraperitoneal, $12 \mathrm{~h}$ before the sacrifice of the mice $(100 \mu \mathrm{L} \mathrm{of} 6 \mathrm{mg} / \mathrm{mL}$ EdU solution for $20 \mathrm{~g}$ of mouse weight) (Click-iT EdU Imaging Kits Alexa Fluor 594, Thermo Fisher A10044, Paisley, UK). Mice were kept in pathogen-free conditions and all procedures conformed to Italian law (D. Lgs ${ }^{\circ}$ 2014/26, implementation of the 2010/63/UE) and approved by the University of Milan Animal Welfare Body and by the Italian Minister of Health. 


\subsection{Isolation of MPs from Skeletal Muscle}

Fascia of the TA muscles was removed. Muscles were dissociated and digested in RPMI medium containing $0.2 \%$ of collagenase B (Roche Diagnostics $\mathrm{GmbH} 11088815001$ ) at $37^{\circ} \mathrm{C}$ for $1 \mathrm{~h}$ and passed through a $70 \mu \mathrm{m}$ and a $30 \mu \mathrm{m}$ cell strainer. $\mathrm{CD} 45^{+}$cells were isolated using magnetic beads (Miltenyi Biotec 130-052-301) and incubated with FcR blocking reagent (Miltenyi Biotec 130-059-901) for 20 min at $4{ }^{\circ} \mathrm{C}$ in PBS $2 \%$ FBS. Cells were then stained with Ly6C-PE (eBioscience 12-5932) and CD64-APC (BD Pharmingen 558539) antibodies for $30 \mathrm{~min}$ at $4^{\circ} \mathrm{C}$. MPs were analyzed or sorted using a FACS Aria III cell sorter (BD Biosciences) (gating strategy is shown Figure S1). In some experiments, $\mathrm{Ly}_{6 \mathrm{C}}{ }^{+}$and $\mathrm{Ly}_{6} \mathrm{C}^{-}$ MPs were cytospined on starfrost (Knitterglaser, Bielefeld, Germany) slides and immunostained.

\subsection{Histology and Immunofluorescence Analyses}

The fascia of TA muscles was removed and the muscles were frozen in liquid nitrogen-cooled isopentane (VWR) and placed at $-80{ }^{\circ} \mathrm{C}$ until cut. Then, $8 \mu \mathrm{m}$-thick cryosections were stained for hematoxylin-eosin (H\&E) and immunofluorescence. H\&E (Sigma-Aldrich, Saint-Louis, MO 63103, USA) staining was processed according to standard protocols. For immunofluorescence analysis, sections or cells were fixed for 15 min with $4 \%$ paraformaldehyde (except for F4/80 and eMyHC staining). Then, samples were permeabilized with 0.5\% Triton X-100 (Sigma-Aldrich) in PBS for $10 \mathrm{~min}$ and blocked with $4 \%$ BSA (Sigma-Aldrich) in PBS at RT for $1 \mathrm{~h}$. Primary antibodies were incubated $\mathrm{O} / \mathrm{N}$ at $4{ }^{\circ} \mathrm{C}$ in PBS. After three washes of 5 min with PBS, samples were incubated with secondary antibodies (1:500, Jackson Laboratory. Fluorochromes used: 488, 594, 546 and 647) and Hoechst (1:500, Sigma-Aldrich) in PBS for $45 \mathrm{~min}$ at RT, then washed four times for 5 min with PBS and mounted with Fluorescence Mounting Medium (Dako). For Nfix-F4/80 double immunolabeling, cryosections were labelled with antibodies against F4/80 (1:400, Novus Biologicals NB300-605) overnight at $4{ }^{\circ} \mathrm{C}$ and Nfix labelling using (1:200, Novus Biologicals NBP2-15039) the antibody was performed for $2 \mathrm{~h}$ at $37^{\circ} \mathrm{C}$. For EdU-Pax7-laminin immunolabelling, after fixation and permeabilization of muscle sections, we followed the manufacturer's instructions of the Click-iT EdU Imaging Kits Alexa Fluor 594 (Thermo Fisher A10044) to reveal the DNA integrated EdU. Then, for Pax7 immunostaining, antigen retrieval was performed by incubating muscle sections in boiling $10 \mathrm{mM}$ citrate buffer $\mathrm{pH} 6$ for $20 \mathrm{~min}$. Muscle sections were then incubated O/N with Pax7 (1:2, Hybridoma, DSHB, Iowa City, IA 52242, USA) and laminin (1:200, Sigma L9393). The other antibodies used were eMyHC (1:2, Hybridome), MyoD (1:50, Santacruz Biotechnology sc-377460), TNF $\alpha$ (1:50, Abcam ab34839), CCL3 (1:500, Abcam ab32609, Cambridge, UK), iNOS (1:25, Novus Biologicals NB300-605, Centennial, CO 80112, USA), CD163 (1:50, Santacruz Biotechnology sc-33560), CD206 (1:50, Bio-Rad MCA2235GA), TGF $\beta$ (1:100, Abcam ab64715), Arginase I (1:100, Santacruz Biotechnology, Cambridge, UK).

\subsection{Bone Marrow Derived MPs (BMDM) Culture}

Total mouse bone marrow was obtained by flushing femur and tibiae with DMEM. Cells were cultured in DMEM containing 20\% Fetal Bovine Serum (FBS) and 30\% of L929 cell line-derived conditioned medium (enriched in CSF-1) for 6 to 7 days. MPs were polarized using $50 \mathrm{ng} / \mathrm{mL} \mathrm{IFN \gamma}$ (for M1 polarization) (Peprotech \#315-05), $10 \mathrm{ng} / \mathrm{mL}$ IL10 (for M2c polarization) (Peprotech \#210-10), in DMEM (10\% FBS) for 3 days. After washing three times, DMEM serum-free medium was added for $24 \mathrm{~h}$, and supernatants were recovered and centrifuged to obtain macrophage-conditioned medium. For some experiments, cells were directly used for various analyses. In some experiments, DMSO or $10 \mu \mathrm{M}$ of ROCK inhibitor Y27632 (Santacruz sc-3536) was added on MPs.

\subsection{Myogenic Progenitor Cells (mpc) Culture}

Murine WT myoblast progenitor cells (mpcs) were obtained from TA muscle and cultured in DMEM/F12 (Gibco, Paisley, UK), containing 20\% FBS and $2.5 \mathrm{ng} / \mathrm{mL}$ of human FGF-basic (Peptrotech, 100-18B). For the proliferation assay, mpcs were seeded at $10000 \mathrm{cell} / \mathrm{cm}^{2}$ on Matrigel 
(1/10) and incubated for 1 day with macrophage-conditioned medium $+2.5 \%$ FBS. Then, cells were incubated with the anti-Ki-67 antibody (1/50, BD Biosciences 550609). For differentiation assay, mpcs were seeded at 30000 cell/ $\mathrm{cm}^{2}$ on Matrigel (diluted 1/10 in DMEM/F12) and incubated for 3 days with macrophage-conditioned medium containing $2 \%$ horse serum. Then, cells were incubated with a pan-myosin antibody (1:2, Hybridoma).

\subsection{Phagocytosis Assay}

Mpcs were labelled using the CellVue Claret Far Red kit (Sigma-Alrich MinClaret) by following the manufacturer's instructions (Sigma-Aldrich) and treated with staurosporin at $5 \mu \mathrm{M}$ for $4 \mathrm{~h}$, in order to induce apoptosis. M1 and M2c polarized MPs were incubated with apoptotic mpcs at a 1:3 ratio for $30 \mathrm{~min}$ at $4{ }^{\circ} \mathrm{C}$ or $6 \mathrm{~h}$ or $16 \mathrm{~h}$ at $37^{\circ} \mathrm{C}$. After three PBS washings, MPs were detached using trypsin and a cell scraper and cells were labelled with a CD64-APC (BD Pharmingen 558539) and analyzed by flow cytometry using a FACS Aria III cell sorter (BD Biosciences). The double-positive cells $\left(\mathrm{CD}_{4}{ }^{+} /\right.$Far Red ${ }^{+}$cells) were phagocytic MPs, whereas the CD64 ${ }^{+} /$Far Red $^{-}$cells were nonphagocytic MPs. To exclude MPs that have bound, but not ingested, apoptotic cells, we subtracted the percentage of double-positive cells observed at $4{ }^{\circ} \mathrm{C}$ from the value observed at $37^{\circ} \mathrm{C}$. In some experiments, MPs were treated with $1 \mu \mathrm{g} / \mathrm{mL}$ of cytochalasin D (Sigma-Aldrich C8273), $45 \mathrm{~min}$ before adding apoptotic mpcs, and with the added mpcs.

\subsection{Lentiviral Transduction}

BMDM from WT mice were transduced with a lentivirus carrying a scrambled sequence or a shNfix [27]. Transduction was performed in suspension (in DMEM 20\% FBS), at a MOI of 10 and in the presence of Polybrene $(8 \mu \mathrm{g} / \mathrm{mL}$, Sigma-Aldrich). After $\mathrm{O} / \mathrm{N}$ incubation, the medium was changed and cells were treated with puromycin $(2 \mu \mathrm{g} / \mathrm{mL}$, Sigma-Aldrich).

\subsection{RNA Extraction and $q R T-P C R$}

RNA was isolated from the sorted apoptotic mpcs, non-phagocyted and phagocyted MPs, by using TRIzol Reagent (Invitrogen 15596026, Bleiswijk, Netherlands), according to the manufacturer's instructions. RNA was quantified using a NanoPhotoneter (Implen). For retro-transcription, $500 \mathrm{ng}$ of RNA was used with the iScript Reverse Transcription Supermix for RT-quantitative qPCR (Bio-Rad 1708840). For qRT PCR, cDNA was diluted 1:10, and $5 \mu \mathrm{L}$ of the diluted cDNA was loaded in a total volume of $20 \mu \mathrm{L}$ (SYBR Green Supermix (Bio-Rad 172-5124) and run on the Bio-RAD CFX Connect Real-Time System. The relative quantification of gene expression was determined by the comparative CT method, and normalized to Cyclophiline A. Primers used were: Nfix for CTGGCTTACTTTGTCCACACTC; Nfix rev CCAGCTCTGTCACATTCCAGAC; Myogenin for CTGGGGACCCCTGAGCATTG; Myogenin rev ATCGCGCTCCTCCTGGTTGA; Cyclo A for GTGACTTTACACGCCATAATG; Cyclo A rev ACAAGATGCCAGGACCTGTAT.

\subsection{Protein Extraction and Western Blot}

Protein extracts were obtained from cultured MPs lysed using RIPA buffer (10 mM Tris- $\mathrm{HCl}$ pH 8.0, 1 mM EDTA, 1\% Triton-X, 0.1\% sodium deoxycholate, $0.1 \%$ sodium dodecylsulphate (SDS), $150 \mathrm{mM} \mathrm{NaCl}$, in deionised water), plus protease and phosphatase inhibitors for $30 \mathrm{~min}$ on ice. Then, samples were centrifuged at $11.000 \times \mathrm{g}$ for $10 \mathrm{~min}$ at $4{ }^{\circ} \mathrm{C}$, and the supernatants collected for protein quantification (DC Protein Assays Bio-Rad 5000111). $40 \mu \mathrm{g}$ protein of each sample were denatured at $95{ }^{\circ} \mathrm{C}$ for $5 \mathrm{~min}$ using SDS PAGE sample-loading buffer $(100 \mathrm{mM}$ Tris pH 6.8, 4\% SDS, 0.2\% bromophenol blue, 20\% glycerol, $10 \mathrm{mM}$ dithiothreitol) and loaded into 8\% SDS acrylamide gels. After electrophoresis, the protein was blotted into nitrocellulose membranes (Protran nitrocellulose transfer membrane; Whatman) for $2 \mathrm{~h}$ at $70 \mathrm{~V}$ at $4{ }^{\circ} \mathrm{C}$. Membranes were then blocked for $1 \mathrm{~h}$ with $5 \%$ milk in Tris-buffered saline, plus $0.02 \%$ Tween20 (Sigma-Aldrich). Membranes were incubated with the primary antibodies $\mathrm{O} / \mathrm{N}$ at $4{ }^{\circ} \mathrm{C}$, using the following antibodies: rabbit anti-Nfix (1:1000, 
Novus Biologicals NBP2-15039), mouse anti-vinculin (1:2500, Sigma-Aldrich V9131), rabbit anti-MYPT1 phosphorylated in Thr696 (1:500, SantaCruz Biotechnology sc-17556-R), and rabbit anti-Tot MYPT1 (1:500; SantaCruz Biotechnology, H-130). After incubation with the primary antibodies, the membranes were washed 3 times for $5 \mathrm{~min}$ and incubated with the secondary antibodies (1:10,000, IgG-HRP, Bio-Rad) for $45 \mathrm{~min}$ at RT, and then washed again 5 times for $5 \mathrm{~min}$. Bands were revealed using ECL detection reagent (ThermoFisher), with images acquired using the ChemiDoc MP system (Bio-Rad). The Image Lab software was used to measure and quantify the bands of at least three independent western blot experiments. The obtained absolute quantity was compared with the reference band (Vinculin) and expressed in the graphs as normalized volume (Norm. Vol. Int.).

\subsection{Image Acquisition and Quantification}

Images were acquired with an inverted microscope (Leica-DMI6000B) equipped with Leica DFC365FX and DFC400 cameras and 20× and 40× magnification objectives. Necrotic myofibers were defined as pink pale patchy fibers, and phagocyted myofibers were defined as pink pale fibers invaded by basophilic single cells (MPs). For the quantification of CSA, analyses were done on damaged TA, which presented at least $75 \%$ of injured muscle. At least 8 pictures in different fields were taken and at least 500 myofibers were analyzed. For each condition of each experiment, at least 8 fields chosen randomly were counted. The number of labelled MPs or mpcs was calculated using the cell tracker in ImageJ software and expressed as a percentage of total MPs or mpcs. Fusion index was the number of nuclei within myotubes divided by the total number of nuclei.

\subsection{Statistical Analysis}

All data shown in the graph are expressed as mean \pm SEM. All experiments were performed using at least three different cultures or animals in independent experiments. A statistical analysis was performed using two-tailed unpaired Student's t-Test, one-way ANOVA or two-way ANOVA. ${ }^{*} p<0.05 ;{ }^{* *} p<0.01 ;{ }^{* * *} p<0.001$; confidence intervals $95 \%$, alpha level 0.05 .

\section{Results}

\subsection{Nfix is Expressed by Anti-Inflammatory MPs}

To understand if the transcription factor Nfix could be involved in MP function, we first analyzed Nfix expression in MPs during normal muscle regeneration in WT mice. We induced muscle injury by cardiotoxin (CTX) injection in the Tibialis Anterior (TA) and looked at the number of MPs (F4/80 cells) positive for Nfix (Figure 1a). While the number of Nfix-positive MPs was identical between day two (D2) and day four (D4) after injury, we observed an increase of MPs expressing Nfix at D7 after CTX injection (Figure 1a). During muscle regeneration, two populations of MPs are present in the damaged tissue. First, the Ly $6 \mathrm{C}^{+}$pro-inflammatory MPs appear and then, they switch into $\mathrm{Ly}_{6 \mathrm{C}^{-}}$ anti-inflammatory population $[3,9,29,34]$. Thus, we asked if Nfix could be expressed by one subset of MPs. We sorted MPs after CTX injury at different time points and looked at Nfix expression by immunolabelling. We firstly enriched for $\mathrm{CD} 45^{+}$cells (mainly composed of MPs and neutrophils) using magnetic beads and then we used the known markers CD64 and Ly6C to separate the pro-inflammatory $\left(\mathrm{CD}^{+} / \mathrm{Ly}^{+} \mathrm{C}^{+}\right.$cells) to the anti-inflammatory MPs $\left(\mathrm{CD}^{+} / \mathrm{Ly}^{+} \mathrm{C}^{-}\right.$cells) (Figure S1). We observed that the percentage of pro-inflammatory MPs expressing Nfix does not change over the time of the regeneration (Figure $1 \mathrm{~b}$ ). On the contrary, the percentage of $\mathrm{CD}^{+} 4^{+} / \mathrm{Ly}_{6} \mathrm{C}^{-}$cells positive for Nfix always increased over time (Figure 1b). We also isolated BMDMs (bone marrow derived MPs) from WT mice and polarized them in pro- or anti-inflammatory phenotype. We observed that anti-inflammatory MPs express more Nfix compared to pro-inflammatory MPs (Figure 1c). Therefore, we can conclude that in both in vitro and in vivo analyses, Nfix is more expressed by anti-inflammatory MPs. 

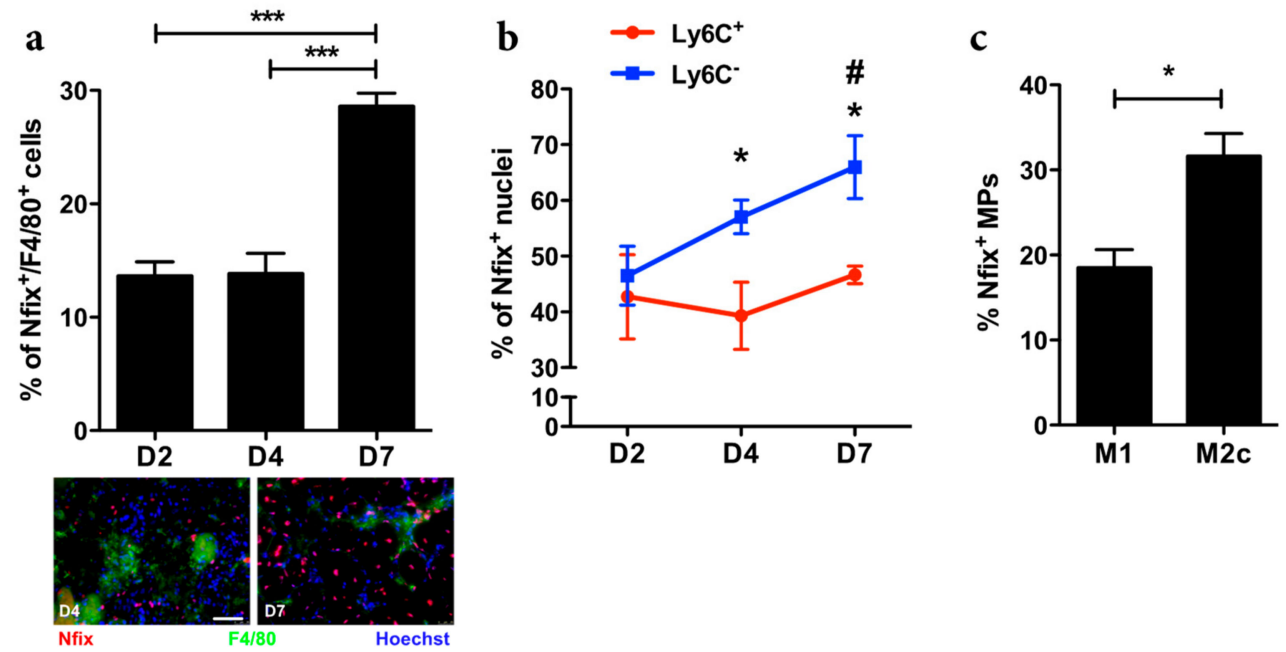

Figure 1. Nfix is mainly expressed by anti-inflammatory MPs. (a) Percentage of F4/80 $\mathrm{MPs}$ positive for Nfix in Tibialis Anterior muscles (TA) of WT mice injected by CTX at D2, D4 and D7, post-injury. Immunostaining for F4/80 (green), Nfix (red) and DAPI (blue) at D4 and D7 after CTX injection; (b) Percentage of $\mathrm{Ly}_{6 \mathrm{C}^{+}}$and Ly6C- sorted MPs positive for Nfix in TA muscles of WT mice injected by CTX at D2, D4 and D7 post-injury; (c) Percentage of Nfix ${ }^{+}$MPs after M1 and M2c polarization (with IFN $\gamma$ and IL10, respectively). ${ }^{*} p<0.05 ;{ }^{* * *} p<0.001$; for $(\mathrm{b}){ }^{*} p<0.05 \mathrm{Ly} 6 \mathrm{C}+\mathrm{vs}$. Ly6C ${ }^{+}$at D4 and D7; $\# p<0.05 \mathrm{Ly} 6 \mathrm{C}^{-} \mathrm{D} 7 \mathrm{vs}$. D2. Results are means $\pm \mathrm{SEM}$ of at least three independent experiments. Scale bar $=50 \mu \mathrm{m}$.

\subsection{Nfix Expression in MPs is Essential for Muscle Regeneration}

We previously demonstrated that Nfix is necessary for the correct differentiation of SCs and, as a consequence, muscle regeneration [27]. In order to understand whether Nfix expression by anti-inflammatory MPs is required for this process, we generated the $\operatorname{LysM}^{\mathrm{CRE}}: \mathrm{Nfix}{ }^{\mathrm{fl} / \mathrm{fl}}$ mice to obtain an animal model deleted for Nfix only in the myeloid line. Once the proper deletion of Nfix in BMDM and $\mathrm{CD} 45^{+}$infiltrated cells two days after muscle injury was verified (Figure S2a), we evaluated the overall phenotype of this new animal model, with particular interest in skeletal muscle morphology at one and two months of life (Figure S2b). No differences were observed between the Nfix ${ }^{\mathrm{fl} / \mathrm{fl}}$ control and the LysM ${ }^{\mathrm{CRE}}: \mathrm{Nfix}{ }^{\mathrm{fl} / \mathrm{fl}}$ mice model in terms of general mouse growth, $\mathrm{TA} / \mathrm{mouse}$ weight and myofiber size (CSA: Cross Sectional Area) (Figure S2b,c). Additionally, we did not observe significant differences in the number of resident MPs expressing Nfix between the Nfix ${ }^{\mathrm{fl} / \mathrm{fl}}$ and the $\mathrm{LysM}^{\mathrm{CRE}}$ : $\mathrm{Nfix}^{\mathrm{fl} / \mathrm{fl}}$ mice (Figure S2d,e). Therefore, the specific deletion of Nfix in MPs in the LysM ${ }^{\mathrm{CRE}}$ : Nfix ${ }^{\mathrm{fl} / \mathrm{fl}}$ mice does not influence the general development of the mice. Notably, in the LysM ${ }^{\mathrm{CRE}}$ : $\mathrm{Nfix}^{\mathrm{fl} / \mathrm{fl}}$ animals resident MPs expressed Nfix similarly to control mice, meaning that the expression of LysM is not required for the establishment of resident MPs.

We then induced muscle injury in control and LysM ${ }^{\mathrm{CRE}}$ : Nfix ${ }^{\mathrm{fl} / \mathrm{fl}}$ mice by CTX injection in TA and we quantified the number of necrotic, phagocyted and regenerating myofibers (Figure 2a,b). Two days after injury, all the myofibers of $\mathrm{Nfix}^{\mathrm{fl} / \mathrm{fl}}$ and LysM ${ }^{\mathrm{CRE}}$ : Nfix $\mathrm{f}^{\mathrm{f} / \mathrm{fl}}$ mice were in necrosis or phagocyted (Figure 2b). At D4 in the control mice, some necrotic and phagocyted myofibers were present, but already $60 \%$ of the fibers were centronucleated (Figure 2b). On the contrary, in the LysM $^{\mathrm{CRE}}: \mathrm{Nfix}{ }^{\mathrm{fl} / \mathrm{fl}}$ mice, we observed a significant decrease in the percentage of centronucleated myofibers $(-31 \%)$ (Figure $2 b)$. While at D7, almost all myofibers were in regeneration in the control mice, the LysM ${ }^{\mathrm{CRE}}: \mathrm{Nfix}{ }^{\mathrm{fl} / \mathrm{fl}}$ mice still exhibited an increase of the percentage of necrotic and phagocyted myofibers and a decrease of centronucleated myofibers $(+282 \%,+150 \%$ and $-30 \%$ respectively), suggesting a delay in the process of muscle regeneration in the absence of Nfix (Figure 2b). We also quantified the CSA of myofibers at D14 and D28 after CTX injury and, in both cases, we observed a decrease of the caliber of myofibers in the LysM ${ }^{\mathrm{CRE}}: \mathrm{Nfix}{ }^{\mathrm{fl} / \mathrm{fl}}$ compared to the $\mathrm{Nfix}^{\mathrm{fl} / \mathrm{fl}}$ mice, due to 
a decrease of the number of big myofibers and an increase of small myofibers (Figure S3a and Figure 2c). These results demonstrated that the expression of Nfix by MPs is necessary for the proper process of muscle regeneration upon acute injury.

a
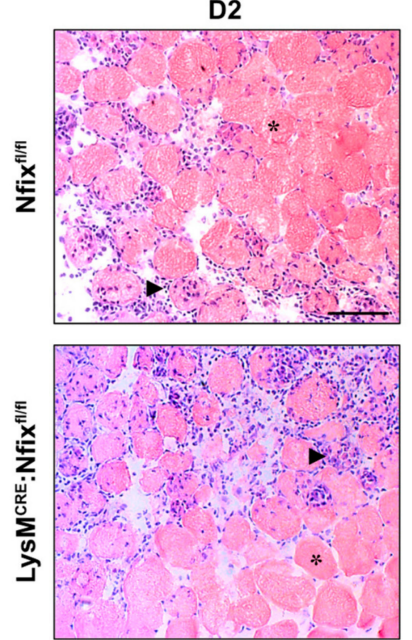

b

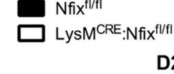

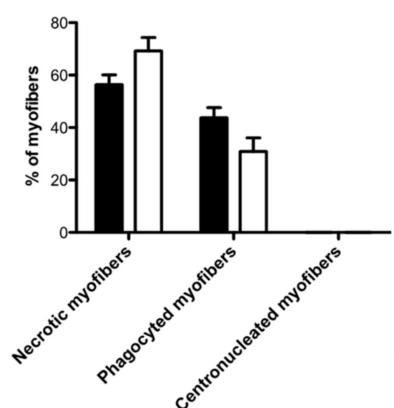

c
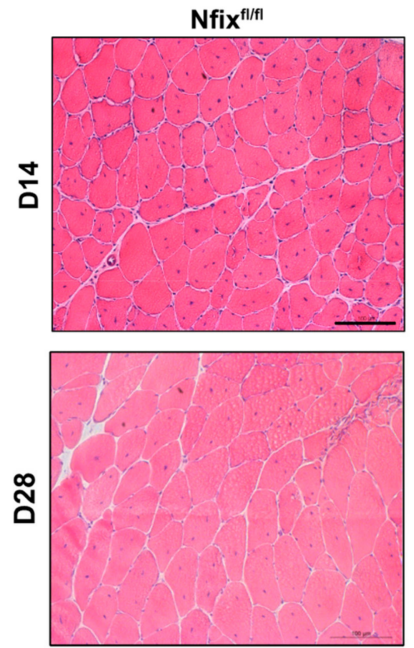

D4
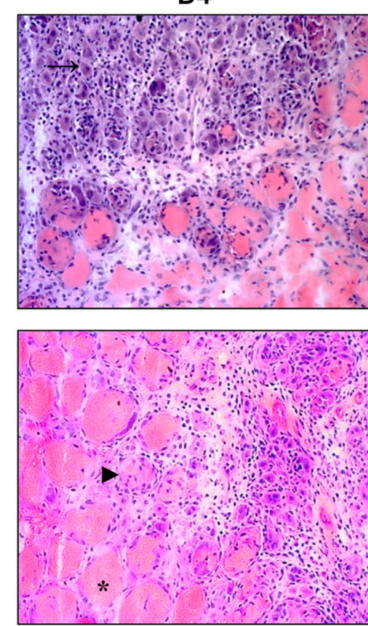

D4

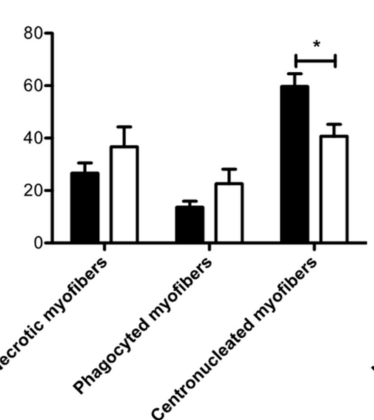

LysM ${ }^{\text {CRE }}: \mathrm{Nfix}^{\mathrm{fl/f1}}$
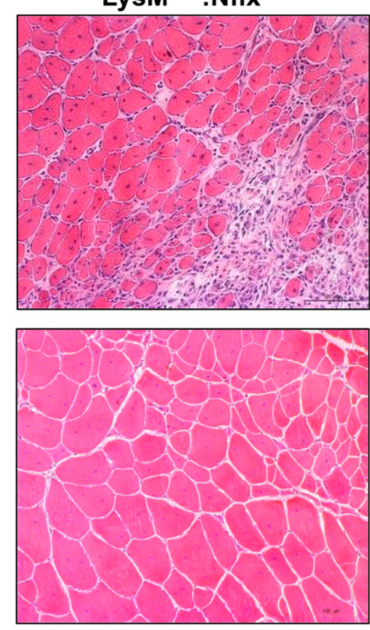

D7
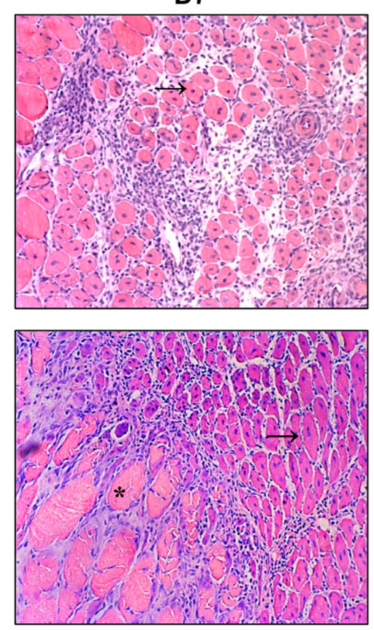

D7
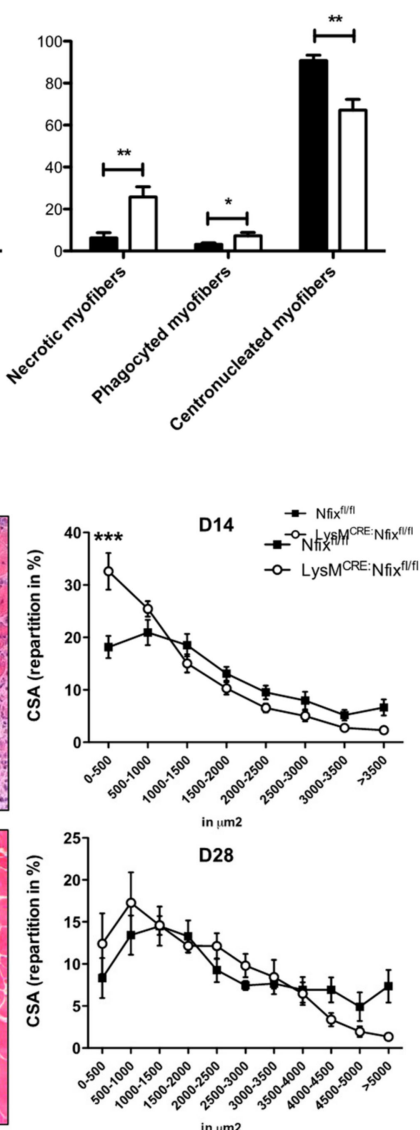

Figure 2. Lack of Nfix in MPs induces a delay of skeletal muscle regeneration. (a) Hematoxylin-eosin staining of $\mathrm{Nfix}{ }^{\mathrm{fl} / \mathrm{fl}}$ and LysM ${ }^{\mathrm{CRE}}: \mathrm{Nfix} \mathrm{fl}^{\mathrm{fl} / \mathrm{fl}} \mathrm{TA}$ muscles injected by CTX at D2, D4, D7 postinjury; (b) Quantification of necrotic (asterisk), phagocyted (arrowhead) and centrally-nucleated (arrow) myofibers, expressed as percentage out of total myofibers; (c) Hematoxylin-eosin staining of Nfix $\mathrm{fl}^{\mathrm{fl}}$ 
and LysM ${ }^{\mathrm{CRE}}: \mathrm{Nfix}{ }^{\mathrm{fl} / \mathrm{fl}} \mathrm{TA}$ muscles injected by CTX at D14 and D28 postinjury and repartition in percentage of the cross-sectional area (CSA). ${ }^{*} p<0.05,{ }^{* *} p<0.01,{ }^{* * *} p<0.001$. Results are means \pm SEM of at least three independent experiments. Scale bar $=100 \mu \mathrm{m}$.

\subsection{Nfix is Required for MP Phenotypical Switch In Vivo and In Vitro}

Defects of muscle regeneration due to MP dysfunction are usually linked to a defect of phenotype acquisition $[8,9,35,36]$. Thus, we looked at the switch from pro- to anti-inflammatory phenotype after CTX injury at different time points by FACS (Figure 3a). First, we did not observe any differences in neutrophils and MPs infiltration between the two mouse models at all time points analyzed (Figure S3b). At two days after injury, a majority of $\mathrm{Ly} 6 \mathrm{C}^{+}$pro-inflammatory MPs was observed in the control Nfix ${ }^{\mathrm{fl} / \mathrm{fl}}$ mice (Figure 3b). Then, at D4 and D7, the ratio between $\mathrm{Ly}_{6 \mathrm{C}^{+}} / \mathrm{Ly} 6 \mathrm{C}^{-} \mathrm{MPs}$ decreased due to the switch from pro- to anti-inflammatory phenotype (Figure $3 \mathrm{~b}$ ). Interestingly, the ratio between $\mathrm{Ly} 6 \mathrm{C}^{+} / \mathrm{Ly} \mathrm{C}^{-}$ in the LysM ${ }^{\mathrm{CRE}}: \mathrm{Nfix} \mathrm{fl}^{\mathrm{fl} / \mathrm{fl}}$ mice was always higher compared to the $\mathrm{Nfix}^{\mathrm{fl} / \mathrm{fl}}$ control, meaning that Nfix is necessary for the switch from the pro- to anti-inflammatory phenotype (Figure $3 b$ ).

We also silenced Nfix in WT BMDM (Bone Marrow Derived MPs) by using a lentiviral vector carrying a small hairpin RNA targeting Nfix (shNfix), or a scrambled sequence as a control (shScramble) [27]. The decrease of Nfix expression in shNfix infected MPs was confirmed by qRT-PCR (Figure S3c). We polarized transduced MPs in pro-inflammatory (M1) and anti-inflammatory (M2c) phenotype (with IFN- $\gamma$ and IL-10, respectively), and we looked at the expression of several proand anti-inflammatory markers by immunofluorescence. As expected, M1 shScramble MPs expressed significantly more $\mathrm{TNF} \alpha$, iNOS and $\mathrm{CCl} 3$ pro-inflammatory markers than M2c shScramble MPs (Figure 3c). Conversely, M2c shScramble MPs expressed more ArgI, TGF $\beta$, CD163 and CD206 anti-inflammatory markers than M1 shScramble MPs (Figure 3c). Interestingly, in the absence of Nfix we observed an increase of pro-inflammatory markers (except for $\mathrm{CCl}$ ) in MPs polarized to M2c phenotype (Figure 3c). We also observed a decrease of MPs positive for anti-inflammatory markers in the polarized M2c MPs lacking Nfix (Figure 3b). These results clearly show that Nfix is necessary for the proper adoption of an anti-inflammatory phenotype and that, without Nfix, MPs remain in a pro-inflammatory status.

\subsection{Nfix is Required for Macrophage Function on Mpcs In Vivo and In Vitro}

Since depending on their phenotype MPs act differentially on WT myogenic progenitor cells (mpcs), we set experiments of conditioned medium (CM) coming from pro- or anti-inflammatory MPs [37]. We added CM coming from BMDM derived from $\mathrm{Nfix}^{\mathrm{fl} / \mathrm{fl}}$ and $\mathrm{LysM}^{\mathrm{CRE}}$ : $\mathrm{Nfix}^{\mathrm{fl} / \mathrm{fl}}$ mice on proliferating or differentiating mpcs. We looked at mpc proliferation by mean of Ki67 staining and at their differentiation by the quantification of the fusion index. As expected, CM coming from M1 Nfix $x^{\mathrm{fl} / \mathrm{fl}}$ MPs stimulated the proliferation of mpcs, while M2c CM had no effect (Figure 4a and Figure S4a). Similarly, CM coming from M1 LysM ${ }^{\mathrm{CRE}}$ :Nfix ${ }^{\mathrm{fl} / \mathrm{fl}} \mathrm{MPs}$ stimulated at the same extent the proliferation of mpcs (Figure 4a and Figure S4a). Interestingly, CM from M2c LysM ${ }^{\mathrm{CRE}}: \mathrm{Nfix}{ }^{\mathrm{fl} / \mathrm{fl}} \mathrm{MPs}$ stimulated the proliferation of mpcs as M1 CM does (Figure 4a and Figure S4b), whereas CM coming from both $\mathrm{Nfix}^{\mathrm{fl} / \mathrm{fl}}$ and LysM ${ }^{\mathrm{CRE}}: \mathrm{Nfix}{ }^{\mathrm{fl} / \mathrm{fl}} \mathrm{M} 1 \mathrm{MPs}$ had no effect on the fusion index of mpcs (Figure $4 \mathrm{~b}$ and Figure S4b). While M2c Nfix ${ }^{\mathrm{fl} / \mathrm{fl}}$ MPs CM increased the fusion of mpcs compared to M1 Nfix ${ }^{\mathrm{fl} / \mathrm{fl}}$ $\mathrm{CM}$, the CM from M2c LysM ${ }^{\mathrm{CRE}}$ :Nfix ${ }^{\mathrm{fl} / \mathrm{fl}}$ MPs lost its pro-fusion effect (Figure $4 \mathrm{~b}$ and Figure $\mathrm{S} 4 \mathrm{~b}$ ). We also investigated if the lack of Nfix in MPs affects myogenic cells in vivo. To quantify mpcs proliferation, we injured, using CTX, the TA of both $\mathrm{Nfix}^{\mathrm{fl} / \mathrm{fl}}$ and $\mathrm{LysM}^{\mathrm{CRE}}: \mathrm{Nfix} x^{\mathrm{fl} / \mathrm{fl}}$ mice and we injected EdU $12 \mathrm{~h}$ before sacrifice. The proliferation of SCs (EdU $/ \mathrm{Pax}^{+}$cells) was identical between the two models at all the time points analyzed (Figure S4c). On the contrary, the percentage of $\mathrm{EdU}^{+} / \mathrm{MyoD}^{+}$ cells at D2 and D4 was higher in the LysM ${ }^{\mathrm{CRE}}: \mathrm{Nfix}{ }^{\mathrm{fl} / \mathrm{fl}}$ mice compared to $\mathrm{Nfix} \mathrm{fl}^{\mathrm{fl} / \mathrm{ll}}$ mice (Figure $4 \mathrm{c}$ ). To quantify the number of newly formed myofibers, we performed an immunofluorescence for eMyHC (embryonic Myosin Heavy Chain) on both injured mouse models (Figure 4d). At D4, the percentage of myofibers positive for $\mathrm{eMyHC}$ was around $80 \%$, meaning that almost all myofibers were formed de 
novo (Figure $4 \mathrm{~d}$ ). At D7, 20\% of the $\mathrm{Nfix}{ }^{\mathrm{fl} / \mathrm{fl}}$ myofibers were positive for the eMyHC and only $6.5 \%$ at D14 (Figure 4d). On the contrary, at D7 and D14 we observed an increase in the number of eMyHC ${ }^{+}$ myofibers in the LysM ${ }^{C R E}: N f i x{ }^{\mathrm{fl} / \mathrm{fl}}$ mice ( $34.7 \%$ and $15.8 \%$ respectively) (Figure $4 \mathrm{~d}$ ). To conclude, Nfix is necessary to MPs to adopt an anti-inflammatory phenotype and, consequently, function. The defect of the phenotypical switch due to the absence of Nfix results in a persistence of pro-inflammatory MPs in the injured muscle, leading to a continuous proliferation of $\mathrm{MyoD}^{+}$cells. The absence of anti-inflammatory MPs induces a delay in the differentiation of new myofibers and, therefore, in the proper muscle regeneration.
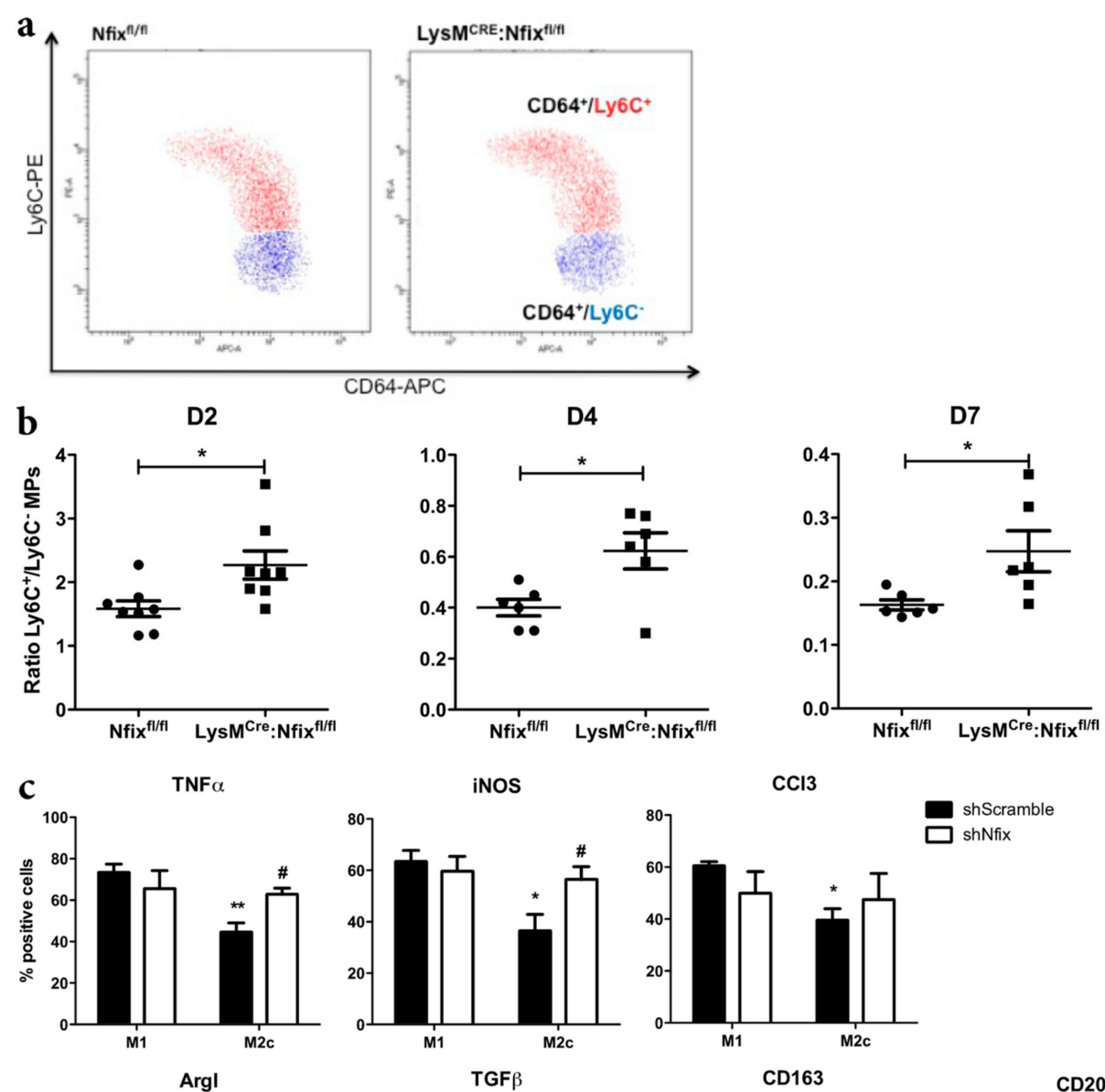

shScramble
shNfix
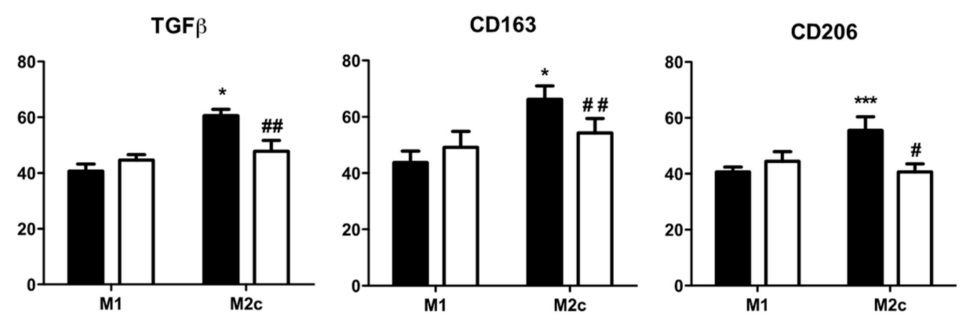

Figure 3. MPs lacking Nfix are unable to adopt an anti-inflammatory phenotype in vivo and in vitro. (a) Representative FACS (Fluorescence-Activated Cell Sorting) gate of pro- and anti-inflammatory $\mathrm{CD}^{+} 4^{+} \mathrm{MP}$ populations in TA of $\mathrm{Nfix}^{\mathrm{fl} / \mathrm{fl}}$ and $\mathrm{LysM}^{\mathrm{CRE}}: \mathrm{Nfix}{ }^{\mathrm{fl} / \mathrm{fl}}$ mice at D2 after CTX injection.

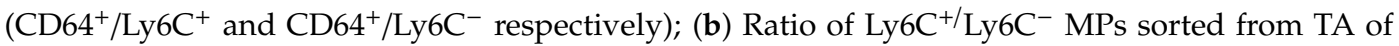
$\mathrm{Nfix}^{\mathrm{fl} / \mathrm{fl}}$ and LysM ${ }^{\mathrm{CRE}}: \mathrm{Nfix}{ }^{\mathrm{fl} / \mathrm{fl}}$ mice at D2, D4 and D7, after CTX injection; (c) WT BMDM (Bone Marrow Derived Macrophages) were transduced by shScramble and shNfix lentiviral vectors and then polarized into M1 and M2c MPs with IFN $\gamma$ and IL10 treatment, respectively. MPs were immunolabeled for pro-inflammatory markers (TNF $\alpha$, iNOS and CCl3) and anti-inflammatory markers (ArgI, TGF $\beta$, CD163 and CD206). The number of positive cells is expressed as percentage out of total cells. ${ }^{*} p<0.05$; 
** $p<0.01 ;{ }^{* * *} p<0.001$ vs. shScramble M1 MPs. ${ }^{\#} p<0.05,{ }^{\# \#} p<0.01,{ }^{\# \#} p<0.001$ vs. shScramble M2c MPs. Results are means \pm SEM of at least three independent experiments.

a
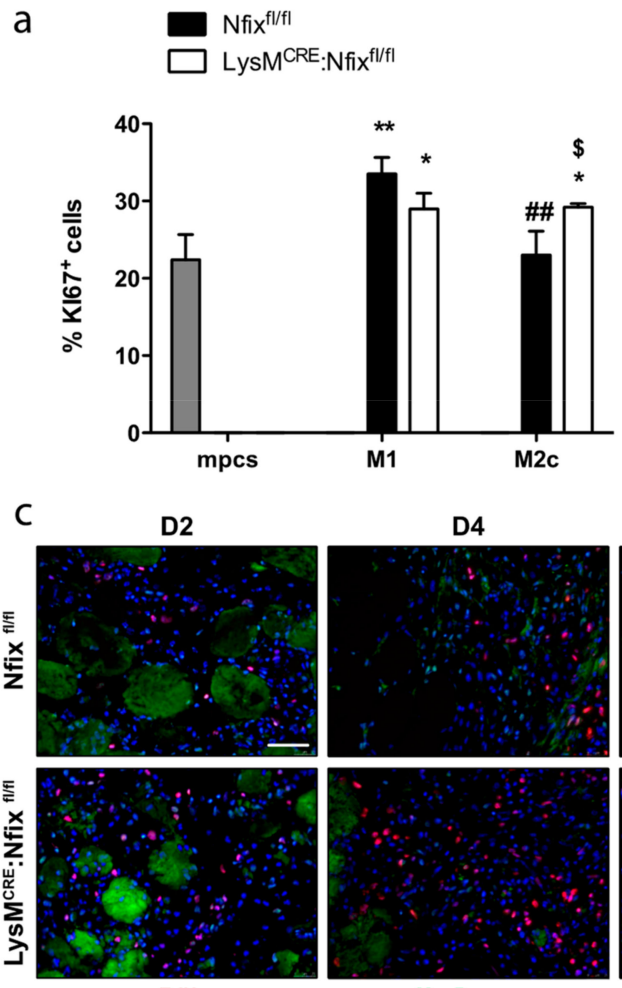

EdU

d
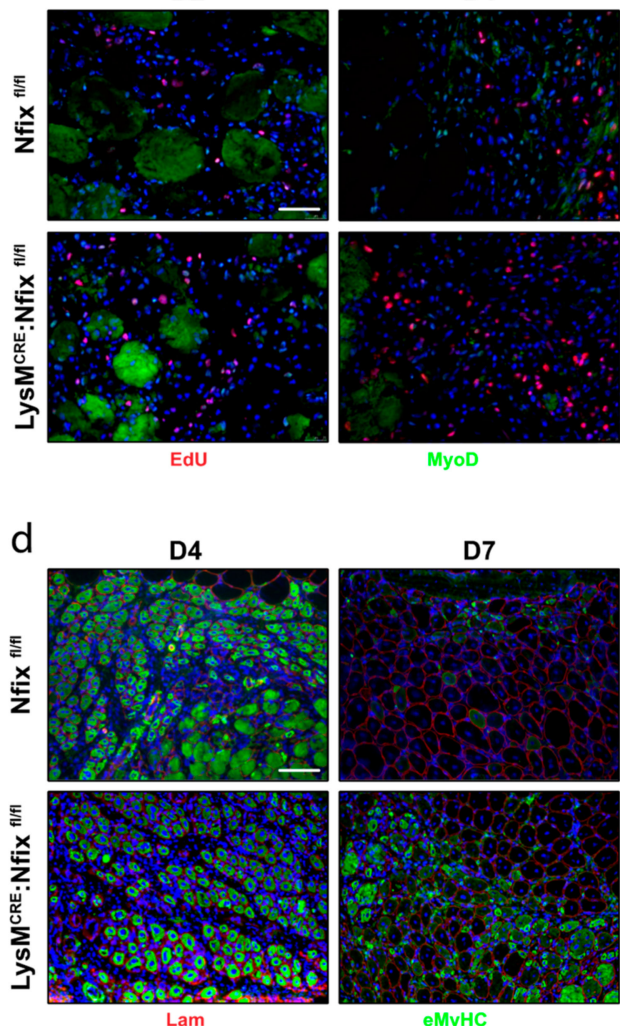

D7

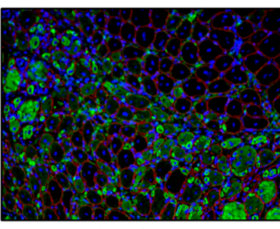

eMyHC b
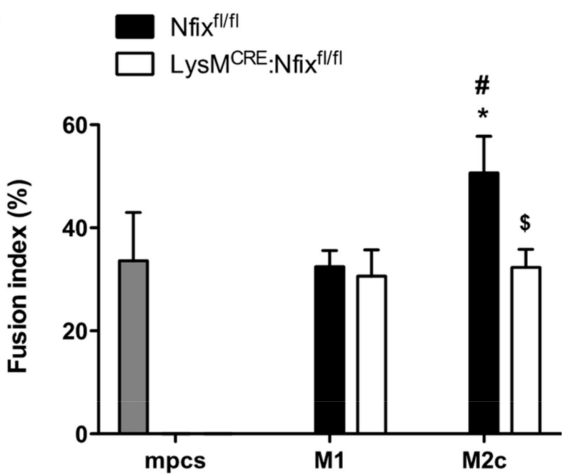

D7

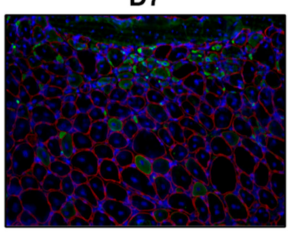

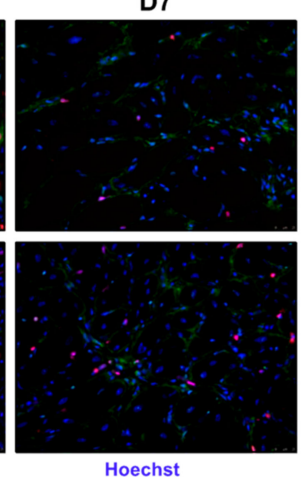

D14

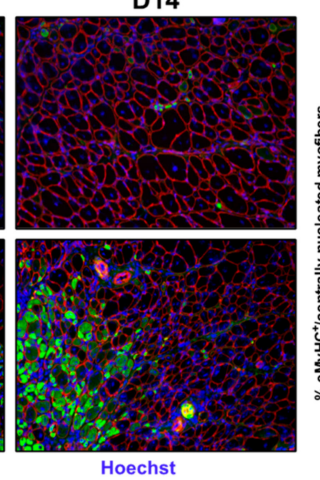

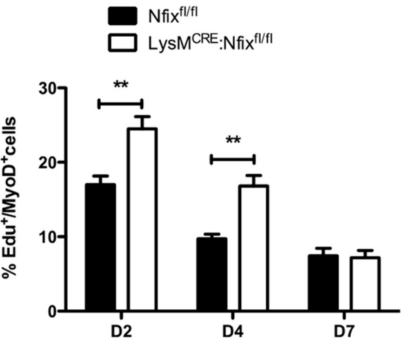

Lysix Lys/fll $^{\text {CRE: }}$ Nfix flffl

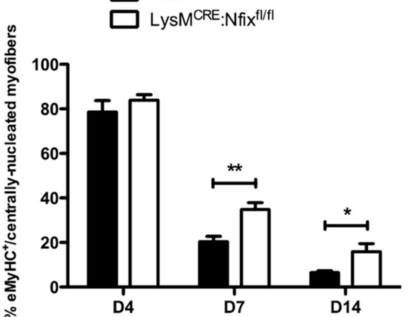

Figure 4. M2 MPs lacking Nfix display M1 MP features on myoblasts in vitro and in vivo. (a) Conditioned medium of M1 or M2c polarized Nfix ${ }^{\mathrm{fl} / \mathrm{fl}}$ and $\mathrm{LysM}^{\mathrm{CRE}}: \mathrm{Nfix}{ }^{\mathrm{fl} / \mathrm{fl}}$ BMDM was added on mpcs, and after $24 \mathrm{~h}$, mpc proliferation was measured as a percentage of Ki67 ${ }^{+}$cells; (b) Conditioned medium of M1 or M2c polarized Nfix ${ }^{\mathrm{fl} / \mathrm{fl}}$ and LysM ${ }^{\mathrm{CRE}}: \mathrm{Nfix}{ }^{\mathrm{fl} / \mathrm{fl}}$ BMDM was added on mpcs and after $72 \mathrm{~h}$, mpcs fusion index was calculated after sarcomeric MyHC staining (\% of $\mathrm{MyHC}^{+}$nuclei into myotubes out of the total nuclei). ${ }^{*} p<0.05,{ }^{* *} p<0.01$ vs. mpcs. ${ }^{\#} p<0.05$, ${ }^{\# \#} p<0.01$ versus M1 Nfix ${ }^{\mathrm{fl} / \mathrm{fl}}$. ${ }^{\$} \mathrm{p}<0.05$ versus same Nfix ${ }^{\mathrm{fl} / \mathrm{fl}}$ polarization; (c) Immunostaining for EdU (red), MyoD (green) and Hoechst (blue) of $\mathrm{Nfix}^{\mathrm{fl} / \mathrm{fl}}$ and LysM ${ }^{\mathrm{CRE}}$ :Nfix ${ }^{\mathrm{fl} / \mathrm{fl}} \mathrm{TA}$ injected by CTX, at D2, D4 and D7 post-injury and quantification of $\mathrm{EdU}^{+} / \mathrm{MyoD}^{+}$cells. EdU was injected in $\mathrm{Nfix}{ }^{\mathrm{fl} / \mathrm{fl}}$ and $\mathrm{LysM}^{\mathrm{CRE}}: \mathrm{Nfix} \mathrm{fl}^{\mathrm{fl} f \mathrm{l}}$ mice $8 \mathrm{~h}$ before sacrifice; (d) Immunostaining for Lam (red), eMyHC (green) and Hoechst (blue) of $\mathrm{Nfix}^{\mathrm{fl} / \mathrm{fl}}$ and LysM ${ }^{\mathrm{CRE}}: \mathrm{Nfix}{ }^{\mathrm{fl} / \mathrm{fl}} \mathrm{TA}$ injected by CTX, at D4, D7 and D14 post-injury and quantification of $\mathrm{eMyHC}^{+} /$centrally-nucleated myofibers. ${ }^{*} p<0.05,{ }^{* *} p<0.01$ Results are means $\pm \mathrm{SEM}$ of at least three independent experiments. Scale bar $=50 \mu \mathrm{m}$. 


\subsection{Phagocytosis Induces the Expression of Nfix}

It has been shown in literature that the phagocytosis of apoptotic cells is the process driving the switch from pro- to anti-inflammatory phenotype, and several studies have demonstrated that MPs presenting a switch defect have a decrease of phagocytic capacity $[8,9,35,38]$. So, we decided to investigate whether the phagocytosis is altered in LysM $^{\mathrm{CRE}}: \mathrm{Nfix}^{\mathrm{fl} / \mathrm{fl}}$ MPs compared to Nfix ${ }^{\mathrm{fl} / \mathrm{fl}}$ MPs. Primary mpcs previously labelled with CellVue-647 were induced to apoptosis and added on Nfix ${ }^{\mathrm{fl} / \mathrm{fl}}$ or LysM ${ }^{\mathrm{CRE}}: \mathrm{Nfix}{ }^{\mathrm{fl} / \mathrm{fl}}$ MPs. After $6 \mathrm{~h}$ in culture, we used a CD64 antibody to discriminate MPs from mpcs: apoptotic mpcs are CellVue- $647^{+} / \mathrm{CD} 64^{-}$, non-phagocytic MPs are CellVue- $647^{-} / \mathrm{CD} 64^{+}$ and phagocytic MPs are CellVue- $647^{+} / \mathrm{CD}^{+} 4^{+}$(Figure S5a). Surprisingly, we did not observe any difference in the phagocytic capacity of $\mathrm{Nfix}^{\mathrm{fl} / \mathrm{fl}}$ and LysM ${ }^{\mathrm{CRE}}: \mathrm{Nfix} \mathrm{fl}^{\mathrm{fl} / \mathrm{fl}} \mathrm{MPs}$ (Figure 5a). Interestingly, while Nfix ${ }^{\mathrm{fl} / \mathrm{fl}} \mathrm{MPs}$ in contact with apoptotic mpcs adopted an anti-inflammatory phenotype (Figure S5b), LysM $^{\mathrm{CRE}}:$ Nfix ${ }^{\text {fl/fl }}$ MPs failed to switch from a pro- to anti-inflammatory phenotype (Figure S5c). Thus, we hypothesized that phagocytosis could induce Nfix expression. To answer to this question, we did the same experiment of phagocytosis using WT MPs and we sorted MPs according to their phagocytic capability (phagocytic and non-phagocytic WT MPs, respectively) (Figure 5b). To verify that no mpcs were sorted with MPs, we first analyzed the expression of myogenin in apoptotic mpcs and in both non-phagocytic and phagocytic MPs. Apoptotic mpcs highly expressed myogenin compared to non-phagocytic and phagocytic MPs and no differences in myogenin expression was observed between the two populations of MPs (Figure S5d). Interestingly, we observed an increase of Nfix expression and MPs positive for Nfix in phagocytic MPs compared to the non-phagocytic ones (Figure 5b). On the contrary, treatment of MPs with cytochalasin D (an inhibitor of phagocytosis) prevents the increase of Nfix positive MPs (Figure $5 \mathrm{c}$ and Figure S5e).

We recently demonstrated that the inhibition of the RhoA-ROCK1 pathway induces Nfix expression in fetal myoblasts and numerous studies have shown that the inhibition of RhoA-ROCK1 increases the phagocytosis, while its stimulation prevents phagocytosis [39-43]. Thus, we treated WT MPs with Y27632, an inhibitor of ROCK1, and after $1 \mathrm{~h}$ of treatment, the phosphorylation of ROCK1-target Mypt decreased, meaning that the inhibition of RhoA-ROCK1 pathway was effective (Figure S5e). After $16 \mathrm{~h}$ of treatment, Y27632-treated WT MPs exhibited an increase of Nfix protein (Figure 5d); most importantly, this increase led to a reduction of pro-inflammatory markers and an increase of anti-inflammatory markers (Figure 5e). On the contrary, this switch through an anti-inflammation phenotype did not occur in LysM ${ }^{\mathrm{CRE}}: \mathrm{Nfix}{ }^{\mathrm{fl} / \mathrm{fl}}$ MPs treated with Y27632 (Figure 5f). These results show that RhoA-ROCK-dependent phagocytosis induces the expression of Nfix, which in turn is necessary to promote the phenotypical switch of MPs from pro- to anti-inflammatory.
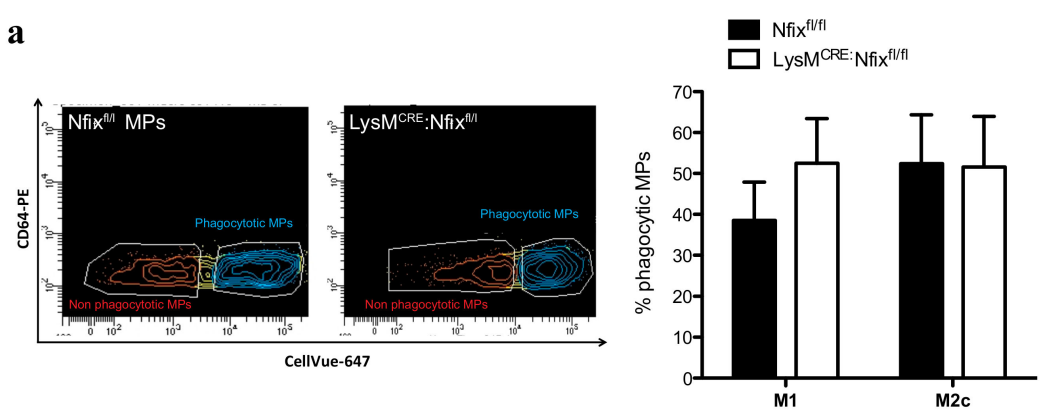

Figure 5. Cont. 
b

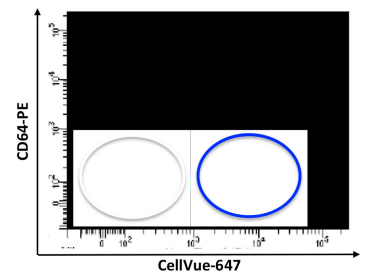

d
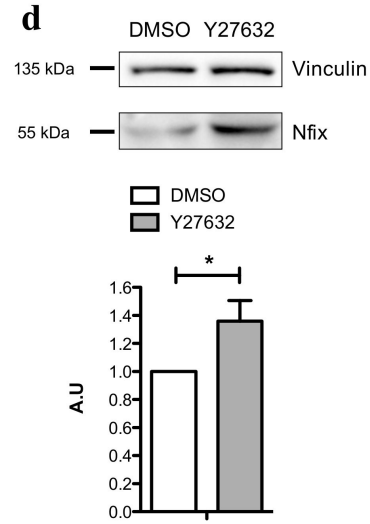

$\square$ No phagocytotic MPs

Phagocytotic MPs

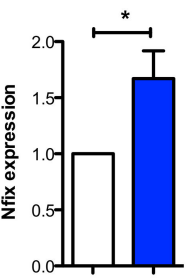

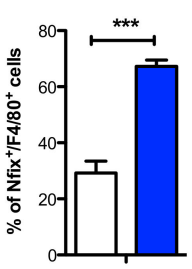

c $\square$ mpcs
$\square$ mpcs + CytoD

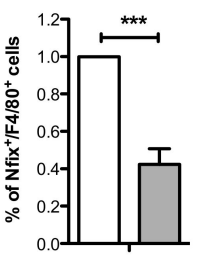

e $\square$ DMSO
$\square$ Y 27632
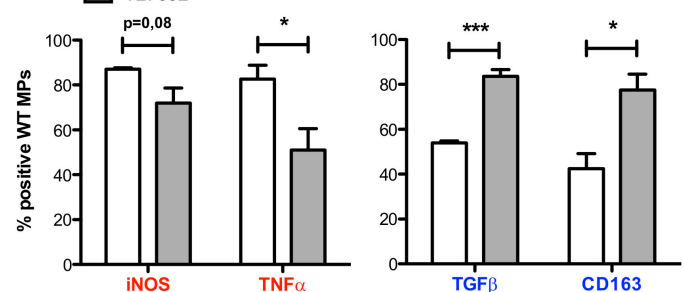

f

$\square$ DMSO
$\square$ Y27632
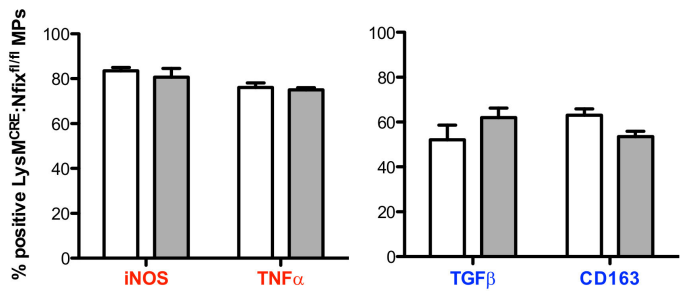

Figure 5. Nfix is expressed after phagocytosis and drive MP phenotypical switch. (a) Phagocytosis assay of M1 and M2c Nfix ${ }^{\mathrm{fl} / \mathrm{fl}}$ and LysM ${ }^{\mathrm{CRE}}: \mathrm{Nfix}{ }^{\mathrm{fl} / \mathrm{fl}}$ MPs cocultured $8 \mathrm{~h}$ with apoptotic mpc. Representative FACS gate of phagocytotic M2c Nfix ${ }^{\mathrm{fl} / \mathrm{fl}}$ and $\mathrm{LysM}^{\mathrm{CRE}}: \mathrm{Nfix}^{\mathrm{fl} / \mathrm{fl}} \mathrm{MPs}\left(\mathrm{CD} 64^{+}\right.$CellVue $\left.{ }^{+}\right)$and percentage of phagocytotic M1 and M2c MPs coming from Nfix ${ }^{\mathrm{fl} / \mathrm{fl}}$ and LysM ${ }^{\mathrm{CRE}}: \mathrm{Nfix} \mathrm{fl}^{\mathrm{fl} / \mathrm{fl}} \mathrm{BMDM} ;(\mathbf{b})$ WT MPs were cocultured $16 \mathrm{~h}$ with apoptotic mpcs. Representative FACS gate of non-phagocytotic $\left(\mathrm{CD} 64^{+} \mathrm{CellVue}^{-}\right)$ and phagocytotic $\left(\mathrm{CD} 64^{+} \mathrm{CellVue}{ }^{+}\right)$WT MPs. Quantification of Nfix expression realized by RT-qPCR on sorted non-phagocytotic and phagocytotic WT MPs and quantification of MPs positive for Nfix $\left(\mathrm{Nfix}^{+} / \mathrm{F} 4 / 80^{+}\right)$realized by IF on non-phagocytotic and phagocytotic WT MPs; (c) WT MPs were cocultured for $16 \mathrm{~h}$ with apoptotic mpcs, with or without addition of Cytochalasin D. Quantification of

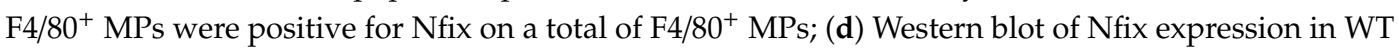
MPs treated with DMSO (Dimethyl sulfoxide) or Y27632 for $16 \mathrm{~h}$ and quantification. Vinculin was used to normalize; (e) WT MPs were treated with DMSO or Y27632 for $16 \mathrm{~h}$ and were immunolabeled for pro-inflammatory markers (iNOS and TNF $\alpha$ ) and anti-inflammatory markers (TGF $\beta$ and CD163). The number of positive cells is expressed as percentage out of total cells; (f) LysM $^{\mathrm{CRE}}: \mathrm{Nfix}{ }^{\mathrm{fl} / \mathrm{fl}}$ MPs were treated with DMSO or Y27632 for $16 \mathrm{~h}$ and were immunolabeled for pro-inflammatory markers (iNOS and TNF $\alpha$ ) and anti-inflammatory markers (TGF $\beta$ and CD163). The number of positive cells is expressed as percentage out of total cells. ${ }^{*} p<0.05,{ }^{* * *} p<0.001$. Results are means \pm SEM of at least three independent experiments.

\section{Discussion}

Skeletal muscle regeneration requires specific temporal steps for the efficacious tissue reconstruction and MPs are the immune cells that are necessary to this process [3,29]. Previous work from our group demonstrated that Nfix null mice exhibit a delay of muscle regeneration, due to a defect of SC differentiation [27]. In this study, we show that the transcription factor Nfix is also expressed by MPs and that mice lacking Nfix in the myeloid lineage have defects in muscle regeneration upon acute injury. We observed that Nfix is preferentially expressed by anti-Ly6C ${ }^{-}$MPs and that its expression increases in time with the progression of the regenerative process. Using an shNfix 
strategy, we observed that M2c MPs silenced for Nfix express higher levels of pro-inflammatory markers (TNF $\alpha$ and Cox2), while they express lower levels of anti-inflammatory markers (CD163, CD206, ArgI and TGF $\beta$ ) than polarized M2c control MPs. Importantly, we observed in vitro that LysM $^{\mathrm{CRE}}: \mathrm{Nfixf}{ }^{\mathrm{l} / \mathrm{fl}} \mathrm{M} 2 \mathrm{c}$ MPs act as M1 MPs on myogenic cells: they stimulate myogenic proliferation and are unable to sustain myogenic differentiation. These two features also occur in vivo since without

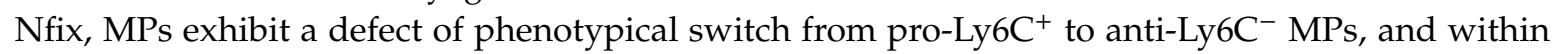
the injured muscle, there is a persistence of myoblast $\left(\mathrm{MyoD}^{+}\right.$cells) proliferation and a delay of newly formed myofibers (eMyHC ${ }^{+}$myofibers). Previous studies showed that the temporal window of the phenotype skewing is a critical step of an effective regeneration. The switch defect $[8,35,36]$ or early appearance of anti-inflammatory MPs impairs muscle regeneration [9]. In line with this evidence, the impairment in the acquisition of an anti-inflammatory phenotype in MPs lacking Nfix leads to a muscle regenerative delay.

So far, the function of Nfix was mainly analyzed in myogenic and neural cells during both development and adult life $[25,27,44-46]$. Recently, Nfix was also shown to play a positive role in the survival of hematopoietic stem and progenitor cells (HSPC) [47], but also to be involved in the fate decision between early B lymphopoiesis and myelopoiesis from blood HSPC [48]. During development, yolk sac gives rise to tissue resident MPs and fetal liver to HSPC, from which blood monocytes and damaged-infiltrating MPs are derived [49]. In our experiments, no differences in the number of infiltrating MPs between control and $\mathrm{LysM}^{\mathrm{CRE}}: \mathrm{Nfix}{ }^{\mathrm{fl} / \mathrm{fl}}$ mice were observed, meaning that the delay observed is due to a defect of macrophage features within the damaged muscle, but not in terms of failed HSPC development.

Little is known about Nfix up-stream regulation, but recently our laboratory identified ERK and RhoA-ROCK1 pathways as, respectively, positive and negative regulators of Nfix expression in pre-natal muscle development. The inhibition of RhoA-ROCK1 induces Nfix expression, promoting myoblasts fusion which is a reflect of myogenesis progression [39]. In MPs, the inhibition of the RhoA-ROCK1 pathway increases the clearance of dead cell phagocytosis, while the constitutive activation of RhoA reduces their phagocytic capacity [40,41]. Importantly, phagocytosis is the process responsible for the induction of the pro- to anti-phenotypical switch in MPs. While numerous studies investigated the mechanisms involved in the progression or inhibition of phagocytosis, how apoptotic cells attract MPs and how MPs recognize them is still unknown [10,14,15,50,51]. In our study, phagocytosis of LysM ${ }^{C R E}: \mathrm{Nfix}{ }^{\mathrm{fl} / \mathrm{fl}} \mathrm{MPs}$ was not impaired compared to control cells. We observed that upon phagocytosis, MPs exhibit an increase in Nfix expression and, conversely, the inhibition of phagocytosis, by using the inhibitor of actin polymerization cytochalasin D, prevents Nfix expression. The stimulation of phagocytosis using the ROCK1 inhibitor Y27632 increases Nfix protein, therefore decreasing pro-inflammatory markers and increasing anti-inflammatory markers in WT MPs. On the contrary, either after phagocytosis or after ROCK1 inhibitor treatment, we observed that MPs lacking Nfix do not have a decrease of pro-inflammatory markers and an increase of anti-inflammatory markers. Thus, the inhibition of the RhoA-ROCK1 pathway induces phagocytosis, leading to Nfix expression that, in turn, drives the MP phenotypical switch.

This study is particularly relevant in light of the recent role for Nfix in muscular dystrophies (MDs)[28]. We indeed demonstrated that the lack of Nfix in two different dystrophic animal models improves both morphological and functional parameters associated to the disease, by promoting a more oxidative musculature and by slowing down muscle regeneration [28]. Different studies have shown that the improvement of dystrophies correlates with a decrease of MPs infiltration [30,32]. While MPs are necessary for muscle regeneration upon acute injury, they are deleterious in the case of chronic injury. Indeed, in muscle myopathies, as in several chronic injured pathologies, MPs are at the origin of fibrosis $[4,32,52,53]$. In the context of acute injury regeneration, pro-inflammatory MPs secrete TNF $\alpha$ that stimulates myoblast proliferation and fibroblast apoptosis, whereas anti-inflammatory MPs secrete TGF $\beta$ that promotes myoblast fusion, but also fibroblast proliferation $[29,54]$. In muscular dystrophies, numerous studies demonstrated that the fibrosis establishment is linked to an over-activation of 
the TGF $\beta$ pathway that stimulates collagen expression by fibroblasts, and in a dystrophic context, more than $75 \%$ of MPs express TGF $\beta$ [54-62]. Thus, in muscle tissue, MPs closely interact with fibroblasts, promoting normal matrix reformation upon acute injury and fibrosis in chronic injury. With this study, we identified Nfix as a new actor of MPs, demonstrating that Nfix is the link between phagocytosis and the phenotypical switch, a necessary step for the resolution of inflammation and tissue repair. Increasing knowledge about signals and factors controlling MP phenotype and, consequently, functions, will help us to understand and control their function in fibrotic pathologies.

Supplementary Materials: The following are available online at http://www.mdpi.com/2073-4409/9/3/708/s1, Figure S1: Gating strategy to isolate MPs from CTX-injured muscles. Figure S2: Characterization of the LysM ${ }^{C R E}: N f i x{ }^{f / f / f l}$ mice. Figure S3: CSA quantification, NT and MPs infiltration in Nfix ${ }^{\mathrm{fl} / \mathrm{fl}}$ and $\mathrm{LysM}^{\mathrm{CRE}}$ :Nfix ${ }^{\mathrm{fl} / \mathrm{fl}}$ mice after CTX injury and Nfix silencing in WT BMDM. Figure S4: In vitro proliferation and differentiation assay. Proliferation of Pax ${ }^{+}$cells after CTX injury. Figure S5: Phagocytosis strategy, inhibition and stimulation.

Author Contributions: Conceptualization, M.S. and G.M.; methodology, M.S., M.L., C.B. and S.A.; validation, M.S., M.L., C.B. and S.A.; formal analysis, M.S.; investigation, M.S., M.L., C.B, G.R. and S.A.; resources, G.M.; data curation, M.S.; writing — original draft preparation, M.S.; writing—-review and editing, G.M.; visualization, M.S.; supervision, G.M.; project administration, G.M.; funding acquisition, G.M. All authors have read and agreed to the published version of the manuscript.

Funding: This research was funded by the European Community, ERCStG2011 (RegeneratioNfix 280611) and the Association Française contre les Myopathies AFM-Telethon (Grant number 20002).

Acknowledgments: We thank Richard Gronostajski for the kind exchange of information and animal models. We are also grateful to Bénédicte Chazaud and Rémi Mounier for helpful discussions and the exchange of animal models.

Conflicts of Interest: The authors declare no conflict of interest.

\section{References}

1. Duffield, J.S.; Forbes, S.J.; Constandinou, C.M.; Clay, S.; Partolina, M.; Vuthoori, S.; Wu, S.; Lang, R.; Iredale, J.P. Selective depletion of macrophages reveals distinct, opposing roles during liver injury and repair. J. Clin. Invest. 2005, 115, 56-65. [CrossRef] [PubMed]

2. Shechter, R.; Miller, O.; Yovel, G.; Rosenzweig, N.; London, A.; Ruckh, J.; Kim, K.W.; Klein, E.; Kalchenko, V.; Bendel, P.; et al. Recruitment of Beneficial M2 Macrophages to Injured Spinal Cord Is Orchestrated by Remote Brain Choroid Plexus. Immunity 2013, 38, 555-569. [CrossRef] [PubMed]

3. Arnold, L.; Henry, A.; Poron, F.; Baba-Amer, Y.; Van Rooijen, N.; Plonquet, A.; Gherardi, R.K.; Chazaud, B. Inflammatory monocytes recruited after skeletal muscle injury switch into antiinflammatory macrophages to support myogenesis. J. Exp. Med. 2007, 204, 1057-1069. [CrossRef] [PubMed]

4. Wynn, T.A.; Vannella, K.M. Macrophages in Tissue Repair, Regeneration, and Fibrosis. Immunity 2016, 44, 450-462. [CrossRef]

5. Vannella, K.M.; Wynn, T.A. Mechanisms of Organ Injury and Repair by Macrophages. Annu. Rev. Physiol. 2017, 79, 593-617. [CrossRef]

6. Chazaud, B. Macrophages: Supportive cells for tissue repair and regeneration. Immunobiology 2014, 219 172-178. [CrossRef]

7. Saclier, M.; Cuvellier, S.; Magnan, M.; Mounier, R.; Chazaud, B. Monocyte/macrophage interactions with myogenic precursor cells during skeletal muscle regeneration. FEBS J. 2013, 280, 4118-4130. [CrossRef]

8. Mounier, R.; Théret, M.; Arnold, L.; Cuvellier, S.; Bultot, L.; Göransson, O.; Sanz, N.; Ferry, A.; Sakamoto, K.; Foretz, M.; et al. AMPK $\alpha 1$ regulates macrophage skewing at the time of resolution of inflammation during skeletal muscle regeneration. Cell Metab. 2013, 18, 251-264. [CrossRef]

9. Perdiguero, E.; Sousa-Victor, P.; Ruiz-Bonilla, V.; Jardí, M.; Caelles, C.; Serrano, A.L.; Muñoz-Cánoves, P. p38/MKP-1-regulated AKT coordinates macrophage transitions and resolution of inflammation during tissue repair. J. Cell Biol. 2011, 195, 307-322. [CrossRef]

10. Lemke, G. How macrophages deal with death. Nat. Rev. Immunol. 2019, 19, 539-549. [CrossRef]

11. Elliott, M.R.; Ravichandran, K.S. The Dynamics of Apoptotic Cell Clearance. Dev. Cell 2016, 38, 147-160. [CrossRef] [PubMed] 
12. Xiao, Y.Q.; Freire-de-Lima, C.G.; Schiemann, W.P.; Bratton, D.L.; Vandivier, R.W.; Henson, P.M. Transcriptional and Translational Regulation of TGF- $\beta$ Production in Response to Apoptotic Cells. J. Immunol. 2008, 181, 3575-3585. [CrossRef] [PubMed]

13. Johann, A.M.; Barra, V.; Kuhn, A.M.; Weigert, A.; Von Knethen, A.; Brüne, B. Apoptotic cells induce arginase II in macrophages, thereby attenuating NO production. FASEB J. 2007, 21, 2704-2712. [CrossRef] [PubMed]

14. Hochreiter-Hufford, A.; Ravichandran, K.S. Clearing the dead: Apoptotic cell sensing, recognition, engulfment, and digestion. Cold Spring Harb. Perspect. Biol. 2013, 5. [CrossRef]

15. Freeman, S.A.; Grinstein, S. Phagocytosis: Receptors, signal integration, and the cytoskeleton. Immunol. Rev. 2014, 262, 193-215. [CrossRef]

16. Tidball, J.G.; Wehling-Henricks, M. Damage and inflammation in muscular dystrophy: Potential implications and relationships with autoimmune myositis. Curr. Opin. Rheumatol. 2005, 17, 707-713. [CrossRef]

17. Dort, J.; Fabre, P.; Molina, T.; Dumont, N.A. Macrophages Are Key Regulators of Stem Cells during Skeletal Muscle Regeneration and Diseases. Stem Cells Int. 2019, 2019. [CrossRef]

18. Farup, J.; Madaro, L.; Puri, P.L.; Mikkelsen, U.R. Interactions between muscle stem cells, mesenchymal-derived cells and immune cells in muscle homeostasis, regeneration and disease. Cell Death Dis. 2015, 6, e1830. [CrossRef]

19. Chazaud, B.; Brigitte, M.; Yacoub-Youssef, H.; Arnold, L.; Gherardi, R.; Sonnet, C.; Lafuste, P.; Chretien, F. Dual and beneficial roles of macrophages during skeletal muscle regeneration. Exerc. Sport Sci. Rev. 2009, 37, 18-22. [CrossRef]

20. Rigamonti, E.; Zordan, P.; Sciorati, C.; Rovere-Querini, P.; Brunelli, S. Macrophage plasticity in skeletal muscle repair. Biomed Res. Int. 2014, 2014. [CrossRef]

21. Biressi, S.; Molinaro, M.; Cossu, G. Cellular heterogeneity during vertebrate skeletal muscle development. Dev. Biol. 2007, 308, 281-293. [CrossRef] [PubMed]

22. Mauro, A. Satellite Cell of Skeletal Muscle Fibers. J. Biophys Biochem Cytol 1961, 9, 493-498. [CrossRef] [PubMed]

23. Dumont, N.A.; Bentzinger, C.F.; Sincennes, M.C.; Rudnicki, M.A. Satellite cells and skeletal muscle regeneration. Compr. Physiol. 2015, 5, 1027-1059. [PubMed]

24. Gronostajski, R.M. Roles of the NFI/CTF gene family in transcription and development. Gene 2000, 249, 31-45. [CrossRef]

25. Messina, G.; Biressi, S.; Monteverde, S.; Magli, A.; Cassano, M.; Perani, L.; Roncaglia, E.; Tagliafico, E.; Starnes, L.; Campbell, C.E.; et al. Nfix Regulates Fetal-Specific Transcription in Developing Skeletal Muscle. Cell 2010, 140, 554-566. [CrossRef] [PubMed]

26. Pistocchi, A.; Gaudenzi, G.; Foglia, E.; Monteverde, S.; Moreno-Fortuny, A.; Pianca, A.; Cossu, G.; Cotelli, F.; Messina, G. Conserved and divergent functions of Nfix in skeletal muscle development during vertebrate evolution. Development 2013, 140, 2443. [CrossRef]

27. Rossi, G.; Antonini, S.; Bonfanti, C.; Monteverde, S.; Vezzali, C.; Tajbakhsh, S.; Cossu, G.; Messina, G. Nfix Regulates Temporal Progression of Muscle Regeneration through Modulation of Myostatin Expression. Cell Rep. 2016, 14, 2238-2249. [CrossRef]

28. Rossi, G.; Bonfanti, C.; Antonini, S.; Bastoni, M.; Monteverde, S.; Innocenzi, A.; Saclier, M.; Taglietti, V.; Messina, G. Silencing Nfix rescues muscular dystrophy by delaying muscle regeneration. Nat. Commun. 2017, 8. [CrossRef]

29. Saclier, M.; Yacoub-Youssef, H.; Mackey, A.L.; Arnold, L.; Ardjoune, H.; Magnan, M.; Sailhan, F.; Chelly, J.; Pavlath, G.K.; Mounier, R.; et al. Differentially activated macrophages orchestrate myogenic precursor cell fate during human skeletal muscle regeneration. Stem Cells 2013, 31, 384-396. [CrossRef]

30. Wehling, M.; Spencer, M.J.; Tidball, J.G. A nitric oxide synthase transgene ameliorates muscular dystrophy in mdx mice. J. Cell Biol. 2001, 155, 123-131. [CrossRef]

31. Kharraz, Y.; Guerra, J.; Mann, C.J.; Serrano, A.L.; Muñoz-Cánoves, P. Macrophage plasticity and the role of inflammation in skeletal muscle repair. Mediators Inflamm. 2013, 2013. [CrossRef] [PubMed]

32. Muñoz-Cánoves, P.; Serrano, A.L. Macrophages decide between regeneration and fibrosis in muscle. Trends Endocrinol. Metab. 2015, 26, 449-450. [CrossRef] [PubMed]

33. Campbell, C.E.; Piper, M.; Plachez, C.; Yeh, Y.-T.; Baizer, J.S.; Osinski, J.M.; Litwack, E.D.; Richards, L.J.; Gronostajski, R.M. The transcription factor Nfix is essential for normal brain development. BMC Dev. Biol. 2008, 8, 52. [CrossRef] [PubMed] 
34. Varga, T.; Mounier, R.; Horvath, A.; Cuvellier, S.; Dumont, F.; Poliska, S.; Ardjoune, H.; Juban, G.; Nagy, L.; Chazaud, B. Highly Dynamic Transcriptional Signature of Distinct Macrophage Subsets during Sterile Inflammation, Resolution, and Tissue Repair. J. Immunol. 2016, 196, 4771-4782. [CrossRef] [PubMed]

35. Ruffell, D.; Mourkioti, F.; Gambardella, A.; Kirstetter, P.; Lopez, R.G.; Rosenthal, N.; Nerlov, C. A CREB-C/EBPbeta cascade induces M2 macrophage-specific gene expression and promotes muscle injury repair. Proc. Natl. Acad. Sci. USA 2009, 106, 17475-17480. [CrossRef] [PubMed]

36. Nie, M.; Liu, J.; Yang, Q.; Seok, H.Y.; Hu, X.; Deng, Z.-L.; Wang, D.-Z. MicroRNA-155 facilitates skeletal muscle regeneration by balancing pro- and anti-inflammatory macrophages. Cell Death Dis. 2016, 7, e2261. [CrossRef]

37. Saclier, M.; Theret, M.; Mounier, R.; Chazaud, B. Effects of macrophage conditioned-medium on murine and human muscle cells: Analysis of proliferation, differentiation, and fusion. Proc. Natl. Acad. Sci. USA 2009, 106, 17475-17480.

38. Arnold, L.; Perrin, H.; de Chanville, C.B.; Saclier, M.; Hermand, P.; Poupel, L.; Guyon, E.; Licata, F.; Carpentier, W.; Vilar, J.; et al. CX3CR1 deficiency promotes muscle repair and regeneration by enhancing macrophage ApoE production. Nat. Commun. 2015, 6, 8972. [CrossRef]

39. Taglietti, V.; Angelini, G.; Mura, G.; Bonfanti, C.; Caruso, E.; Monteverde, S.; Le Carrou, G.; Tajbakhsh, S.; Relaix, F.; Messina, G. RhoA and ERK signalling regulate the expression of the transcription factor Nfix in myogenic cells. Development 2018, 145. [CrossRef]

40. Bros, M.; Haas, K.; Moll, L. Grabbe RhoA as a Key Regulator of Innate and Adaptive Immunity. Cells 2019, 8, 733. [CrossRef]

41. Nakaya, M.; Tanaka, M.; Okabe, Y.; Hanayama, R.; Nagata, S. Opposite effects of Rho family GTPases on engulfment of apoptotic cells by macrophages. J. Biol. Chem. 2006, 281, 8836-8842. [CrossRef] [PubMed]

42. Königs, V.; Jennings, R.; Vogl, T.; Horsthemke, M.; Bachg, A.C.; Xu, Y.; Grobe, K.; Brakebusch, C.; Schwab, A.; Bähler, M.; et al. Mouse Macrophages completely lacking Rho subfamily GTPases (RhoA, RhoB, and RhoC) have severe lamellipodial retraction defects, but robust chemotactic navigation and altered motility. J. Biol. Chem. 2014, 289, 30772-30784. [CrossRef] [PubMed]

43. Kim, S.Y.; Kim, S.; Bae, D.J.; Park, S.Y.; Lee, G.Y.; Park, G.M.; Kim, I.S. Coordinated balance of Rac1 and RhoA plays key roles in determining phagocytic appetite. PLoS ONE 2017, 12. [CrossRef] [PubMed]

44. Harris, L.; Zalucki, O.; Gobius, I.; McDonald, H.; Osinki, J.; Harvey, T.J.; Essebier, A.; Vidovic, D.; Gladwyn-Ng, I.; Burne, T.H.; et al. Transcriptional regulation of intermediate progenitor cell generation during hippocampal development. Development 2016, 143, 4620-4630. [CrossRef]

45. Harris, L.; Dixon, C.; Cato, K.; Heng, Y.H.E.; Kurniawan, N.D.; Ullmann, J.F.P.; Janke, A.L.; Gronostajski, R.M.; Richards, L.J.; Burne, T.H.J.; et al. Heterozygosity for Nuclear Factor One X Affects Hippocampal-Dependent Behaviour in Mice. PLoS ONE 2013, 8. [CrossRef]

46. Fraser, J.; Essebier, A.; Gronostajski, R.M.; Boden, M.; Wainwright, B.J.; Harvey, T.J.; Piper, M. Cell-type-specific expression of NFIX in the developing and adult cerebellum. Brain Struct. Funct. 2017, 222, 2251-2270. [CrossRef]

47. Holmfeldt, P.; Pardieck, J.; Saulsberry, A.C.; Nandakumar, S.K.; Finkelstein, D.; Gray, J.T.; Persons, D.A.; Mckinney-freeman, S. Nfix is a novel regulator of murine hematopoietic stem and progenitor cell survival. Blood 2015, 122, 2987-2997. [CrossRef]

48. O'Connor, C.; Campos, J.; Osinski, J.M.; Gronostajski, R.M.; Michie, A.M.; Keeshan, K. Nfix expression critically modulates early B lymphopoiesis and myelopoiesis. PLoS ONE 2015, 10, 1-15. [CrossRef]

49. Gomez Perdiguero, E.; Klapproth, K.; Schulz, C.; Busch, K.; de Bruijn, M.; Rodewald, H.R.; Geissmann, F. The Origin of Tissue-Resident Macrophages: When an Erythro-myeloid Progenitor Is an Erythro-myeloid Progenitor. Immunity 2015, 43, 1023-1024. [CrossRef]

50. Park, S.Y.; Kim, I.S. Engulfment signals and the phagocytic machinery for apoptotic cell clearance. Exp. Mol. Med. 2017, 49. [CrossRef]

51. Haney, M.S.; Bohlen, C.J.; Morgens, D.W.; Ousey, J.A.; Barkal, A.A.; Tsui, C.K.; Ego, B.K.; Levin, R.; Kamber, R.A.; Collins, H.; et al. Identification of phagocytosis regulators using magnetic genome-wide CRISPR screens. Nat. Genet. 2018, 50, 1716-1727. [CrossRef] [PubMed]

52. Ngambenjawong, C.; Gustafson, H.H.; Pun, S.H. Progress in tumor-associated macrophage (TAM)-targeted therapeutics. Adv. Drug Deliv. Rev. 2017, 114, 206-221. [CrossRef] 
53. Tang, P.M.K.; Nikolic-Paterson, D.J.; Lan, H.Y. Macrophages: Versatile players in renal inflammation and fibrosis. Nat. Rev. Nephrol. 2019, 15, 144-158. [CrossRef] [PubMed]

54. Lemos, D.R.; Babaeijandaghi, F.; Low, M.; Chang, C.-K.; Lee, S.T.; Fiore, D.; Zhang, R.-H.; Natarajan, A.; Nedospasov, S.A.; Rossi, F.M. V Nilotinib reduces muscle fibrosis in chronic muscle injury by promoting TNF-mediated apoptosis of fibro/adipogenic progenitors. Nat. Med. 2015, 21, 786-794. [CrossRef]

55. Ueha, S.; Shand, F.H.W.; Matsushima, K. Cellular and molecular mechanisms of chronic inflammation-associated organ fibrosis. Front. Immunol. 2012, 3, 1-6. [CrossRef]

56. Tan, R.J.; Liu, Y. Macrophage-derived TGF-beta in renal fibrosis: Not a macro- impact after all. Am. J. Physiol. Renal Physiol. 2013, 1-7.

57. Mann, C.J.; Perdiguero, E.; Kharraz, Y.; Aguilar, S.; Pessina, P.; Serrano, A.L.; Muñoz-Cánoves, P. Aberrant repair and fibrosis development in skeletal muscle. Skelet. Muscle 2011, 1, 21. [CrossRef]

58. Vidal, B.; Serrano, A.L.; Tjwa, M.; Suelves, M.; Ardite, E.; De Mori, R.; Baeza-Raja, B.; De Lagrán, M.M.; Lafuste, P.; Ruiz-Bonilla, V.; et al. Fibrinogen drives dystrophic muscle fibrosis via a TGF $\beta /$ alternative macrophage activation pathway. Genes Dev. 2008, 22, 1747-1752. [CrossRef] [PubMed]

59. Tidball, J.G.; Wehling-Henricks, M. Shifts in macrophage cytokine production drive muscle fibrosis. Nat. Med. 2015, 21, 665-666. [CrossRef]

60. Pakshir, P.; Hinz, B. The big five in fibrosis: Macrophages, myofibroblasts, matrix, mechanics, and miscommunication. Matrix Biol. 2018, 68-69, 81-93. [CrossRef]

61. Smith, L.R.; Barton, E.R. Regulation of fibrosis in muscular dystrophy. Matrix Biol. 2018, 68-69, 602-615. [CrossRef] [PubMed]

62. Biernacka, A.; Dobaczewski, M.; Frangogiannis, N.G. TGF- $\beta$ signaling in fibrosis. Growth Factors 2011, 29, 196-202. [CrossRef] [PubMed]

(C) 2020 by the authors. Licensee MDPI, Basel, Switzerland. This article is an open access article distributed under the terms and conditions of the Creative Commons Attribution (CC BY) license (http://creativecommons.org/licenses/by/4.0/). 